\title{
Realtime Facility Management System Framework based on Building Information Modeling and Web of Things
}

\author{
Seongki Lee and Sung-Ah Kim \\ Department of Architecture, Sungkyunkwan University \\ 2066, Seobu-Ro, Jangan-Gu, Suwon, Republic of Korea \\ *Eun-Ho Oh, Eun-Young Shin, Tai-Kyung Kang, and Yoo-Sub Lee, \\ Korea Institute of Construction Technology \\ 1190, Simindae-Ro, Ilsanseo-Gu, Goyang, Republic of Korea \\ (*Corresponding author: uno1988@kict.re.kr)
}




\title{
Real-time Facility Management System Framework based on Building Information Modeling and Web of Things
}

\begin{abstract}
The purpose of this paper is to propose the real-time facility management system framework based on Building Information Model (BIM) and Web of Things. Latest IT such as BIM is rapidly spreading to architecture, civil engineering, engineering and maintenance with the aim of simplifying complex system in buildings and facilities, minimizing operation cost, providing high value services and advancing facility management. In particular, BIM-based management framework design to assist the development of sensor information operation system, which adds convenience to facility maintenance and management, has emerged as a key issue. To this end, this paper proposed conceptual framework for data warehouse, web service and interface that make up real-time facility management system. The paper also produced conceptual methodology on CoRE-based web service platform, mobile device, BIM and WSN mapping.
\end{abstract}

\section{KEYWORDS}

Building Information Model, Web of Things, CAFM, BEMS

\section{INTRODUCTION}

\section{The need to link BIM with information real-time}

Korea's Public Procurement Service requires BIM-based design for construction orders worth more than 50 billion KRW, which is driving BIM technology's rapid spread to architecture, civil engineering, engineering and maintenance business. At the same time, there is a growing global trend towards developing convergence technologies for detecting dynamic environmental changes to respond to frequent earthquakes around the world and for integration with technologies to predict uncertainties. Such convergence between the latest IT and existing building system is driven by the need to reduce operating cost, to simplify the complex system and rely less on highly-skilled manpower to save capital cost and to provide high value service. This is why it is important to design BIM-based management framework to develop a sensor operation system which makes facility management and maintenance more convenient. The integration of building infrastructure and data operation contributes to reduce operating cost and new service opportunities. Session 2 looked into the latest trend of smart building and WSN (Wireless Sensor Network). Session 3 proposed conceptual framework of real-time facility management system, data warehouse, web service and interface, CoRE (Constrained RESTful Environments)-based web service platform in particular, mobile device, BIM and WSN mapping that make up the system and discussed achievement and significance of the study.

\section{RELATED RESEARCH}

\section{Smart buildings and environmental impact}


Smart buildings accompany a series of technologies that monitor buildings, and improve not only safety but also energy efficiency and user convenience. These technologies range from new materials with higher efficiency and Information Communication Technologies (ICTs). ICTs are used in the system to control heating, lighting and ventilation in smart buildings. It also provides the foundation for automatic package and crime prevention control system that automatically turns off PCs, monitors and other devices [3]. Latest study on third-generation smart building system has come as far as learning the building itself through self-learning and optimizing monitoring as well as crime prevention control function [4]. Sensor and sensor network technologies are widely used in smart building applications like HVAC, lights, shading, air conditioning, window/door control, switch, measuring instrument, standard appliances, crime prevention and safety. All contribute to a dramatic energy savings [5].

\section{WSN and Web of Things (WoTs)}

Building management systems in the past mostly relied on wired network, which were costly and suffered from inflexible design. Wireless sensor and actuator network, on the other hand, has many advantages compared to the wired solution [5]. It is much easier to install wireless sensor-actuator network since it does not require wired network, it can independently produce energy and can be operated on a segregated basis for a long time. Wireless solutions like EnOcean Zigbee, Bluetooth, IEEE 802.15.4 and 6LoWPAN (IPv6 over Low power Wireless Personal Area Networks) are gaining great attention for building automation system [11]. Building management system based on integrated management framework can cut down the heavy management cost and simplify the overall work process. Lately, a new technology which enables building in or installing lightweight embedded web server into equipments in buildings and integrating with network via IP-based wireless communications to monitor information on equipments through web is under limelight. Development of software and system capable of controlling equipments to offer the most optimal service to users by converging information measured by each of equipments is also active.

Information flow in the network model can be put under control by managing scattered data. Web's biggest strength comes from its ability to communicate and link between heterogeneous equipments and make service searching and providing easier. Furthermore, application service can be easily developed. Consequently, replacement of communications and service protocol previously defined by each application domain into WoTs paradigm boosts interoperability and accessibility. Also time as well as cost put into application service development can be significantly reduced.

Works to standardize WoTs and M2M (Machine to Machine) are active at the moment and CoAP (Constrained Application Protocol) standardization, which began standardization activity since 2010 as 6LoWPAN's upper application layer protocol, is underway by CoRE working group in IETF (Internet Engineering Task Force) [11]. Also, study on RESTful interface and CoAP, which are two key technologies of WoTs, has become highly robust and this is urgently raising the need to secure related technologies since it is expected to expand markets for homes, buildings, cars, grids, RFID and USN.

Buildings have a lot of different equipments for cooling, heating, ventilation, lighting, disaster prevention and security. And since they all have different communications interface and protocol, a physical interface and protocol converter to ensure a single uniform access to different interfaces is necessary for macroscopic integrated control. This is critical for buildings that have a wide range of equipments for cooling, heating, ventilation, lighting, disaster prevention and security but each equipment group is manually controlled by operator and protocol and communications mode is different by equipment manufacturer. The lack of interoperability as a result is challenging automatic control that is optimized and integrated. Hence, development of an embedded web server/client supporting integrated management of building equipments based on standardized wireless communications and TCP/IP applicable to building equipments and facilities is underway [11]. Wireless arrangement of automatic building control system taking advantage of the embedded web server/client not only saves cost by approximately $30 \%$ compared to wired arrangement but also cuts installation time by more than $60 \%$. 


\section{CONCEPTUAL FRAMEWORK OF REAL-TIME FACILITY MANAGEMENT SYSTEM}

\section{WSN based Facility Management System Framework}

Conceptual framework of real-time facility management system supports the network of sensors and actuators in buildings to provide M2M service as illustrated in Figure 1. Information transmitted from the system and commands from Control Center are processed via Middleware in Sensors and Actuation Networks.

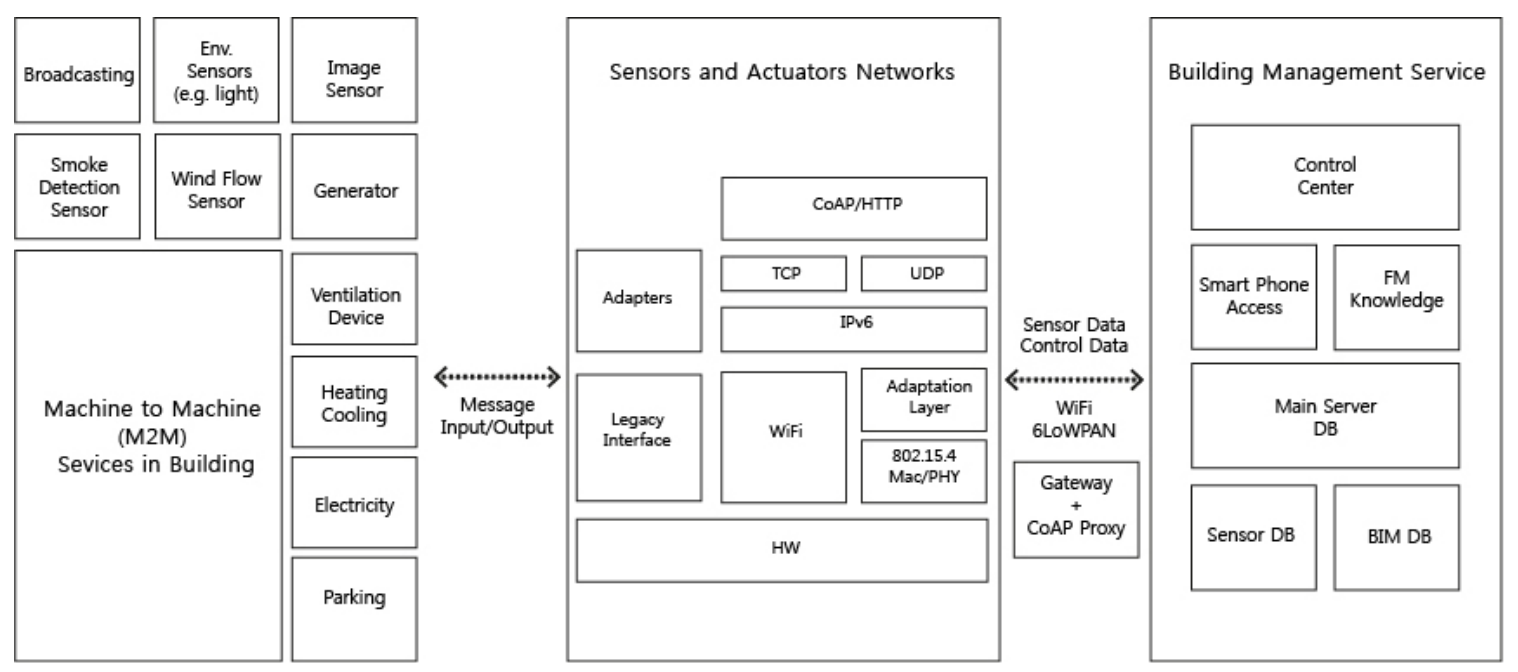

Figure 1 - System framework

Data Warehouse, Web Service and Interface

Data warehouse is designed to manage data collected from multiple sources and keep them in a single storage space for future inquiries and analysis [6]. For example, data warehouse is necessary to store historical data for creating management reports. A separate data warehouse schema is also required to better monitor building performance and collect condition data from sensors to store them in related tables. The schema is designed to connect BIM data with a set of data.

The purpose of web service is to set up a system based on web and component in a single framework. The reason web service is necessary is that, by supporting Service Oriented Architecture (SOA), web service easily provides series of service required to give FM personnel access to information on building performance and management via mobile service. Web service shall be developed in ways to access maintenance work information that was previously processed so that cause of facility failure can be identified, equipment inspected and repair results confirmed or to provide information access to building equipment's performance level during a certain time frame. Communication with all BIM servers is done through service interface. Interface basically refers to Java interface executed on a remote basis and is a group of many methods. Communication with BIM server from a remote PC relies on languageindependent network-based protocols such as SOAP and REST so that it can be processed in all kinds of languages.

\section{CoRE-based Embedded Web Service Platform Technology and Mobile Device}


CoRE-based embedded web service platform technology fulfills WoTs and M2M, which provides access and controls resources in building sensors and control devices via web service interface, and takes advantage of such resources to provide convergence service that fits the situation and user requirements. Implementation of CoRE technology requires REST (Representational State Transfer) web service interface technology and CoAP that can support REST web service interface technology in an embedded environment. RESTful web service, based on HTTP and XML, enables resource access and control via GET/POST/PUT/DELETE method and CoAP is application-level transmission protocol designed to suit environments with poor computing resources and communication.

By nature of the industry, those referred to as mobile technology in FM does not mean that installed devices move on a continuously. Instead, it is attached and fixed to a certain part in a certain component (ex. architectural materials like wall or column). Facility administrator responsible for building management and maintenance specifies certain device requirements as follows [7] - 1. Data stored in the database and web server should be accessible anytime, anywhere. 2. Communication with sensors in building plants should be available. 3. Interoperability with wireless network should be guaranteed. Also, potential challenges such as limited battery power, wireless data transmission, limited processing capacity and limited memory should be identified and prototype should be developed quickly. Development of applications based on iOS or Android platform should be considered and final application can be manufactured with GWT (Google Web Toolkit) [8] capable of supporting broad web browsers and minimizing most of compatibility issues related to web development.

\section{Open BIM and WSN Mapping}

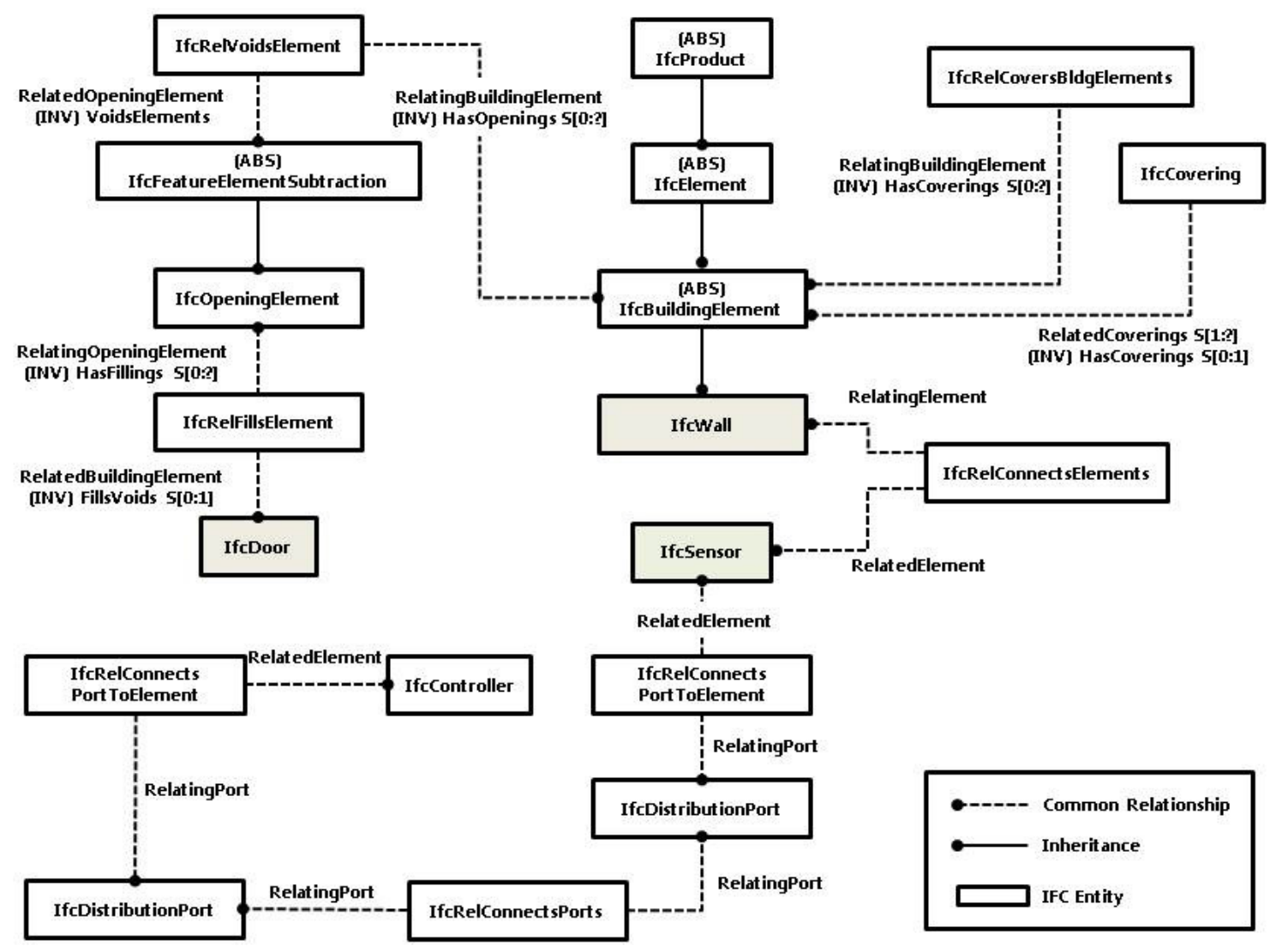

Figure 1 - Relation of building entities, connectivity, and sensor network in IFC [1] 
A study on setting up knowledge and information environment that supports commissioning across BLM (Building Life Cycle Management) to enable real-time monitoring and analysis by integrating physical facility information and virtual information from the FM point of view is underway [10]. BIMserver is mainly used in BIM database [9] and BIMserver open source tool that enables central processing of information in the construction business has been developed [2]. BIMserver adopts modeloriented architecture, which is interpreted via core-object, and support IFC data to be stored in the corresponding database. This allows users to inquire, integrate and filter building information via the tool. Figure 2 shows building structures with building entities, connectivity of space, relation between sensor and controller via authoring tool and management tool. Such relations can contribute to efficient integration with sensor information by structuring DB schema.

\section{CONCLUSIONS}

NIPA's 2010 construction IT convergence technology classification table classifies BIM and sensor information connectivity technology in smart building USN as key technologies. In addition, a technology for real-time connectivity of GIS-BIM linking technology, sensor information and threedimensional spatial information in smart construction operation and management is necessary. This very technology, which is a smart building technology converging building, electricity and electronic and IT, will have a big impact on construction IT convergence industry like IBS and BIM once developed and will evolve into a whole new applied science around component technology around hi-tech sensor system, IT and architectural engineering. Various information about the building itself and others acquired via numerous sensors in a facility and equipment will ensure managing in-building resource effectively and maintaining energy optimally. Development of sensor maintenance hardware/software linked to mobile devices will also become more active.

Development of a sensor maintenance technology based on context resource management linked with BIM system and establishment of a standard information management system linked with national standard BIM system are also expected to gain further momentum. RESTful interface and CoAP protocol technology, which offers easy access to and control of things' functions and data, will hopefully position themselves into key technologies in distributed embedded system and M2M communications. This technology uses the same language as existing web protocol. In other words, it can take advantage of HTML, XML and URI to access and control things' functions and data. Also, the use of web will enable more advanced type of convergence service like browsing, searching and bookmarking.

Development of convergence service engine software technology to design and interpret component-based user-tailored optimal control algorithm aimed at controlling facility optimally based on user behavior/space cognition and maintenance technology is forecasted to transform paradigm of automation system design and integrated monitoring service required for constructing buildings in the future. Finally, WoTs and M2M paradigm will support information exchange among all objects and between objects and humans, which will eventually be utilized to provide convergent service.

\section{ACKNOWLEDGEMENT}

The research presented in this paper is based upon work supported by "BIM/GIS Platform based Construction Project Management Technology." The authors appreciate Korea Institute of Construction Technology for supporting the research fund.

\section{REFERENCES}


[1] Oh, E.-H., Lee, S., Shin, E.-Y., T.-K., and Lee, Y.-S. (2012). A Framework of Real-time Infrastructure Disaster Management System based on the Integration of the Building Information Model and the Sensor Information Model, Journal of KOSHAM 12(6), 007-014

[2] Kim, S.-A., Choe, Y., Jang, M., and Seol, W. (2011). Design Process Visualization System Integrating BIM Data and Performance-Oriented Design Information, Proceedings of ISARC 2011, part 2, 122-127

[3] SMART 2020: Enabling the low carbon economy in the information age, The Climate Group

[4] Sharples, S., Callaghan, V., and Clarke, G., (1999). A multi-agent architecture for intelligent building sensing and control, Sensor Review 19(2), 135-140

[5] Czubak, A., and Wojtanowski, J. (2009) On Applications of Wireless Sensor Networks. In Tkacz, E., and Kapczynski, A. (eds), Internet - Technical Development and Applications. Springer Berlin Heidelberg, 91-99

[6] Immon, W. H. (1992), Building the Data Warehouse, John Wiley and Sons

[7] Campos, J., Jantunen, E., and Prakash, O. (2009). A web and mobile equipment architecture for mobile e-maintenance. The International Journal of Advanced Manufacturing Technology 45 (1-2), 71-80

[8] Chaganti, P., Google Web Toolkit - GWT Java AJAX Programming, Packt Publishing Ltd., 2007

[9] Beetz, J., Van Berlo, L, de Latt, R. and van den Helm, P. (2010). BIMserver.org - An open source IFC model server, Proc. of the CIB-W78 Conference, Cairo, 1-8

[10] Stack, P., Ryan, J., Walsh, B., Quinlan, J., and Menzel, K. (2011), Context-Sensitive Maintenance Management using Mobile Tools, AET 2011, 374-381

[11] Shelby, Z. and Bormann, C. (2009). 6LoWPAN: The wireless embedded Internet, John Wiley \& Sons Ltd. 